\title{
Recurrent Esophageal Adenocarcinoma
}

National Cancer Institute

\section{Source}

National Cancer Institute. Recurrent Esophageal Adenocarcinoma. NCI Thesaurus. Code C8627.

An esophageal adenocarcinoma which has reappeared after a period of remission or after presumed treatment. 